\title{
Orthomolecular Medicine
}

National Cancer Institute

\section{Source}

National Cancer Institute. Orthomolecular Medicine. NCI Thesaurus. Code C61371.

This subcategory refers to products used as nutritional and food supplements (and not covered in other categories). These products are used for preventive or therapeutic purposes. They are usually used in combinations and at high doses. Examples include niacinamide for arthritis and melatonin to prevent breast cancer. 